\title{
BMJ Open Using environmental engineering to increase hand hygiene compliance: a cross-over study protocol
}

\author{
Kelly Ann Schmidtke, ${ }^{1}$ Navneet Aujla, ${ }^{1}$ Tom Marshall, ${ }^{1,2}$ Abid Hussain, ${ }^{3}$ \\ Gerard P Hodgkinson, ${ }^{4}$ Kristopher Arheart, ${ }^{5}$ Joachim Marti, ${ }^{6}$ David J Birnbach, ${ }^{7}$ \\ Ivo Vlaev ${ }^{1}$
}

\begin{abstract}
To cite: Schmidtke KA, Aujla N, Marshall T, et al. Using environmental engineering to increase hand hygiene compliance: a cross-over study protocol. BMJ Open 2017;7:e017108. doi:10.1136/ bmjopen-2017-017108

- Prepublication history and additional material for this paper are available online. To view these files please visit the journal online (http://dx.doi. org/10.1136/bmjopen-2017017108).
\end{abstract}

Received 31 March 2017 Revised 22 June 2017

Accepted 23 June 2017

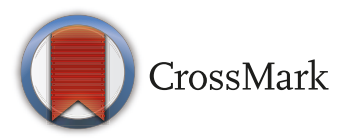

For numbered affiliations see end of article.

Correspondence to Dr Kelly Ann Schmidtke; Kelly.Schmidtke@wbs.ac.uk

\section{ABSTRACT}

Introduction Compliance with hand hygiene

recommendations in hospital is typically less than $50 \%$. Such low compliance inevitably contributes to hospitalacquired infections that negatively affect patients' wellbeing and hospitals' finances. The design of the present study is predicated on the assumption that most people who fail to clean their hands are not doing so intentionally, they just forget. The present study will test whether psychological priming can be used to increase the number of people who clean their hands on entering a ward. Here, we present the protocol for this study.

Methods and analysis The study will use a randomised cross-over design. During the study, each of four wards will be observed during four conditions: olfactory prime, visual prime, both primes and neither prime. Each condition will be experienced for 42 days followed by a 7-day washout period (total duration of trial $=189$ days). We will record the number of people who enter each ward and whether they clean their hands during observation sessions, the amount of cleaning material used from the dispensers each week and the number of hospital-acquired infections that occur in each period. The outcomes will be compared using a regression analysis. Following the initial trail, the most effective priming condition will be rolled out for 3 months in all the wards. Ethics and dissemination Research ethics approval was obtained from the South Central-0xford C Research Ethics Committee (16/SC/0554), the Health Regulatory Authority and the sponsor.

Trial registration number ISRCTN (15397624); Edge ID 86357.

\section{INTRODUCTION}

Over 300000 patients in England suffer a hospital-acquired infection (HAI) annually. ${ }^{1}$ This is a problem in terms of patient safety and hospital finances. ${ }^{23}$ A substantial proportion of these HAIs are avoidable through simple measures, including better hand hygiene. ${ }^{4}$ ${ }^{5}$ Unfortunately, observations suggest that compliance with hand hygiene recommendations is typically less than $50 \% .^{6}$ As human behaviour is at the heart of hand hygiene compliance, interventions informed by

\section{Strength and limitation of this study}

- This study uses a methodologically robust design to assess the effects of novel interventions to improve hand hygiene compliance in hospital.

- The interventions are informed by behavioural psychology theory.

- The study is pragmatic because the setting reflects usual clinical care.

- The primary outcome is hand hygiene compliance at the ward entrance. This is a process measure, and there are many opportunities for hand hygiene beyond ward entrances.

- Although frequency of hospital-acquired infections will be measured, the study is not powered to detect differences in hospital-acquired infections.

suitably rigorous behavioural research may help increase it.

Interventions to change people's behaviour can target the reflective (ie, controlled) and/ or reflexive (ie, automatic) processes posited by dual-process theories. ${ }^{7}$ While the precise nature of these processes is debated, generally it is agreed that reflective processes are slower, more analytic and more controlled, whereas automatic processes are faster and less deliberative..$^{9} 10^{11}$ The question as to whether controlled, automatic or both processes should be targeted to change behaviour depends on a range of factors, including the practicalities of the situation at hand. ${ }^{12} 13$

The present study focuses on interventions that target automatic processes using primes to nudge people entering a ward to clean their hands. ${ }^{14}$ This focus was selected because so many people already know that they should clean their hands in hospital, and all hospital staff receive mandatory training on how to clean their hands. ${ }^{15}$ ${ }^{16}$ Thus, in the absence of personalised feedback, a large educational campaign is 
unlikely to yield large and sustainable effects. ${ }^{17}$ Furthermore, many people who do not clean their hands are likely not doing so intentionally; it is more likely they are simply forgetting. ${ }^{18}$ Placing primes into the hospital environment that nudge more people to clean their hands is a novel way forward. ${ }^{19}$

Priming techniques use existing state-action associations that trigger habitual actions by making those habitual actions more accessible in memory. ${ }^{20}{ }^{21}$ Stimuli referred to as priming cues have been used successfully to activate health-related behaviour. For example, to encourage greater physical activity in one study, participants were asked to make a sentence out of scrambled words such as fit, lean, active and athletic and that resulted in more participants using stairs instead of elevators. ${ }^{22}$ More relevant to the present study, visual and olfactory primes have already been used to increase hand hygiene compliance in two studies. ${ }^{23}{ }^{24}$ The present study aims to extend the findings from these studies.

In the first study, medically trained participants were asked to examine an actor complaining of palpitations. ${ }^{23}$ During the examination, participants' hand hygiene behaviours were monitored using a video surveillance system. Participants were randomly assigned to experience either a standard environment (control group) or a citrus-smelling environment (intervention group). While only $51 \%$ of participants in the standard environment cleaned their hands before interacting with the actor, $80 \%$ of participants in the citrus-smelling environment did.

In the second study, the entry way to an active surgical ward was modified from a standard environment to include either a citrus smell or a picture of someone's eyes above an alcohol gel dispenser. ${ }^{24}$ A researcher recorded in real-time whether people entering the ward used the gel dispenser. While only $15 \%$ of people used the gel dispenser in the standard environment, $47 \%$ did so when the citrus smell was present, and $33 \%$ did so when the picture of someone's eyes was present.

While the findings from previous study in an actual hospital environment are encouraging, the present study addresses some of its limitations. First, the previous study was only conducted in a single surgical ward in the USA; this raises the concern that the previous study's findings might be context specific. To overcome this limitation, the present study will be performed in three non-surgical wards and one surgical ward in the UK.

Second, each prime in the previous study was only active for four non-consecutive days. This raises the concern that the effects found in the previous study do not endure beyond this short time frame. To overcome this limitation, the primes in the present study will each be active for 42 consecutive days.

The present study will also extend the previous study's findings in five important ways. First, whereas the previous study only tested each prime in isolation, the present study will also test the primes' joint effects by presenting them simultaneously. In so doing, our results will be able to suggest whether two primes have greater effects than one.

Second, the previous study only examined the proportion of people using a gel dispenser, whereas the present study will also assess the amount of gel material used from the dispensers. This addition is beneficial because it would be infeasible for our observers to record hand hygiene activity every moment the study is active.

Third, whereas the previous study only assessed the primes' effectiveness in increasing people's use of a gel dispenser, the present study will also assess the primes' effectiveness in increasing people's use of soap dispensers. Observing both is important because although hand rub gels protect against the spread of the infection known as methicillin-resistant Staphylococcus aureus (MRSA), they do not protect against the spread of another type of infection known as Clostridium difficile infection (CDI) ${ }^{25}$

Fourth, whereas the previous study only recorded the gender of the people entering the wards, we will also record their roles at the hospital (eg, doctor or nurse). As hospital staff receive mandatory hand hygiene training, plausibly staff may clean their hands more often than visitors. Interestingly, at least two studies have found that nurses are more likely to clean their hands than doctors. ${ }^{26}{ }^{27}$ A study of hand hygiene behaviour within airport settings suggests that women may be more likely to clean their hands after using the restroom than men. ${ }^{28}$ However, whether the primes affect these different types of people differently is largely unknown.

Fifth, we will compare the number of HAIs that occur in each condition: neither prime, olfactory prime, visual prime and both primes. We acknowledge that the brief duration of the present study may prevent us from finding a significant effect between conditions. To anticipate this potential shortfall, a roll-out of the most effective prime from the initial study is planned for 3 months across all four of the wards incorporated in the initial trail. The change in the number of HAIs that occur in the observed wards over this time, in contrast to those that have occurred over the previous 10 years, will be fed into an economic model to evaluate the cost-effectiveness of the intervention.

\section{OBJECTIVES/HYPOTHESES}

The present study has five objectives.

\section{Objective 1}

To compare the proportion of people who use the gel and soap dispensers when they enter a ward during each of our four conditions (neither prime, olfactory prime, visual prime and both primes) and to assess whether the primes' effects change over time.

\section{Hypothesis 1}

The proportion of people who use the gel and soap dispensers will be greater when the olfactory and/or visual primes are present than when they are not. 


\section{Hypothesis $1 b$}

The proportion of people who use the gel and soap dispensers will be greater when the olfactory and visual primes are presented in combination than when either prime is presented alone.

\section{Objective 2}

To corroborate the findings from objective 1 using the amount of gel and soap materials used from the dispensers.

\section{Hypothesis 2a}

The amount of product used from the gel and soap dispensers will be greater when the olfactory and/or visual primes are present than when they are not.

\section{Hypothesis $2 b$}

The amount of product used from the gel and soap dispensers will be greater when the olfactory and visual primes are presented in combination than when either prime is presented alone.

\section{Objective 3}

To assess the effectiveness of the primes for different types of people; differentiated specifically based on their role at the hospital (doctors, nurses, other hospital staff or visitors) and gender (female or male).

\section{Hypothesis 3a}

The proportion of hospital staff who clean their hands will be greater than the proportion of visitors who clean their hands.

\section{Hypothesis $3 b$}

The proportion of women who clean their hands will be greater than the proportion of men who clean their hands.

\section{Objective 4}

To compare the number of HAIs that occur during the study across our four conditions.

\section{Hypothesis $4 \mathrm{a}$}

There will be fewer HAIs when the olfactory and/or visual primes are present than when they are not.

\section{Hypothesis $4 b$}

There will be fewer HAIs when the olfactory and visual primes are presented in combination than when either prime is presented alone.

\section{Objective 5}

To assess the cost-effectiveness of the interventions in terms of cost per additional percentage of compliance, cost per avoided HAI and cost per quality-adjusted lifeyear (QALY).

\section{Hypothesis 5}

Within the first part of the present study, the effectiveness of the primes can be compared against a control condition wherein there are no primes. Then, the effectiveness of the most promising intervention from the first part will be rolled out in all four wards for 3 months, and the number of HAIs that occur will be compared with the previous 10 years when no primes were active.

Additionally, the cost of our interventions per additional percentage of compliance in the short term will be compared with other previously published results using hand hygiene observations. Then, a modelling exercise expresses the potential long-term cost-effectiveness in terms of cost per HAI avoided and cost per life-year gained and QALY.

\section{Study design}

The study will use a randomised cross-over design, with data collection beginning on 10 March 2017 and ending on 15 September 2017. During the study, each of four wards will be observed during four conditions: (1) neither prime-that is, control, (2) olfactory prime, (3) visual prime and (4) both primes. Following this, the most effective priming condition will be rolled out for 3 months (mid-October 2017 to mid-January 2017) in all four wards.

\section{METHODS}

\section{Study setting}

The present study is sponsored by the University of Warwick and Heart of England NHS Foundation Trust. The study will be conducted at the entrance of four hospital wards selected by the Director of Infection Prevention and Control. All the wards are general wards in that they can take on overflow from other wards, but each ward is specialised in one of the following areas: acute haematology/oncology, admissions, renal medicine and upper gastrointestinal surgery. Note that while the trial takes place in a hospital, it does not fit WHO's definition of a clinical trial and was retrospectively registered at the International Standard Randomised Controlled Trials Number (ISRCTN) registry. ${ }^{29}$

\section{Eligibility criteria}

No participants will be recruited into the study. Any person who walks into the wards while the study is active may have their hand hygiene activity recorded. Walking people below an age of 18 years will be included because it would be infeasible to approach people during observation sessions to ask their age, and the gel and soap material use is automatically recorded. However, during the observation sessions, researchers will not record whether people unable to use the dispensers do; for example, babies in pushchairs and non-ambulatory patients. An information poster about the study will be posted in the atrium of the hospital (online supplementary appendix 1).

\section{Interventions}

A single researcher is named as responsible for the conduct of the study to ensure the interventions are 
active when and where planned. A second researcher will be designated to take over if the named researcher cannot. Before every observation session, the researchers observing will ensure that the primes are in working order and take appropriate action if they are not, for example, call the company providing the scent dispensers.

The olfactory prime will comprise a citrus scent. To display the olfactory primes, the entrance area of each ward will be fitted with a commercial dispenser from ScentDirect. ${ }^{30}$ The olfactory prime will only be dispensed in the wards when scheduled.

The visual prime will comprise a picture of a person's eyes. ${ }^{31}$ To display the visual primes, copies of the picture will be fitted above a gel and soap dispenser in the entrance area of each ward. The visual prime will be printed on an industrial laser printer and laminated. The visual prime will only be displayed in the wards when scheduled.

\section{Outcomes}

Primary outcome

The proportion of people who use the gel dispenser. This outcome relates to objective 1 . It is the outcome measure for which the study was powered based on the effect size found in the previous study in an actual hospital environment. ${ }^{24}$ The number of people who use the gel dispensers when they enter the ward will be the numerator, and the number of people who enter the ward will be the denominator. This will be recorded during the observation sessions.

\section{Secondary outcomes}

The secondary outcomes are being examined for exploratory purposes only.

1. Proportion of people who use the soap dispenser: This outcome relates to objective 1 . The number of people who use the soap dispensers when they enter the ward will be the numerator, and the number of people who enter the ward will be the denominator. These data will be recorded during the observation sessions.

2. The amount of gel dispenser product used: This outcome addresses objective 2. The amount of material used from the gel dispensers will be weighed and recorded by a researcher each Friday.

3. The amount of soap dispenser product used: This outcome addresses objective 2. The amount of material used from the soap dispensers will be weighed and recorded by the researchers each Friday.

4. The number of each type of person entering the wards: This outcome addresses objective 3. During observation sessions, researchers will record whether each person entering the ward appears to be a male or female, and whether they appear to be a doctor, nurse, other staff or visitor.

5. HAIs: This outcome addresses objectives 4 and 5. The number of infections that occur on each ward during the study will be recorded by the Director of Infection Prevention and Control using hospital records. Past census data from the hospital records will be obtained to identify the number of HAIs that have occurred in those wards in the past 10 years. Collecting 10 years of data related to HAI will be useful to better understand long-term trends in HAI in the country and to put the effects of our intervention into perspective.

\section{Timeline of events}

Each ward will experience four conditions. In any given 6-week observation period, one condition will be active in each ward, interspersed with a 1-week break prior to the introduction of a new condition. Each ward will be assigned a condition such that no two wards are in the same condition at the same time, that is, a Latin squares design. ${ }^{32}$ A schematic layout of this schedule is presented in table 1.

During each 6-week observation period, eight separate hour-long observation sessions will be conducted. The researchers conducting observation sessions will observe per a standard operating procedure. Observation sessions will be scheduled to take place on Monday and Wednesday mornings between 9:00-10:00 and 10:3011:30. Whichever time a ward is observed on Monday, it will be observed at the other time on Wednesday. The narrowness of these times was selected to minimise the variation in our data owing to the natural daily variation in hand hygiene compliance. ${ }^{33}$

Only one observer will observe a given ward at a time so that the study causes minimal disruptions to the hospital's day-to-day routines. The observing researchers will switch what wards they observe each week to avoid observer bias affecting the results, for example, a layout of this schedule for the first 2 weeks is in table 2. Two researchers will be primarily responsible for conducting these observation

Table 1 Schedule of intervention conditions

\begin{tabular}{|c|c|c|c|c|c|c|c|}
\hline \multirow[b]{2}{*}{ Ward entrance } & \multicolumn{2}{|l|}{ Phase 1} & \multicolumn{2}{|l|}{ Phase 2} & \multicolumn{2}{|l|}{ Phase 3} & \multirow{2}{*}{$\begin{array}{l}\text { Phase } 4 \\
\text { Weeks } \\
22-27\end{array}$} \\
\hline & $\begin{array}{l}\text { Weeks } \\
1-6\end{array}$ & $\begin{array}{l}\text { Week } \\
7\end{array}$ & $\begin{array}{l}\text { Weeks } \\
8-13\end{array}$ & $\begin{array}{l}\text { Week } \\
14\end{array}$ & $\begin{array}{l}\text { Weeks } \\
15-20\end{array}$ & $\begin{array}{l}\text { Week } \\
21\end{array}$ & \\
\hline Ward B & Control & Control & Both & Control & Olfactory & Control & Visual \\
\hline Ward C & Olfactory & Control & Control & Control & Visual & Control & Both \\
\hline
\end{tabular}


Table 2 Schedule of the observation sessions within the first observation period

\begin{tabular}{|c|c|c|c|c|c|c|}
\hline \multirow[b]{2}{*}{ Ward entrance } & \multicolumn{6}{|l|}{ Week 1} \\
\hline & Observer & Monday & Tuesday & Wednesday & Thursday & Friday \\
\hline Ward A & $x$ & 9:00-10:00 & & $10: 30-11: 30$ & & \\
\hline Ward C & $x$ & 10:30-11:30 & & 9:00-10:00 & & \\
\hline Ward D & $\mathrm{Y}$ & 10:30-11:30 & & 9:00-10:00 & & \\
\hline Ward entrance & & Monday & Tuesday & Wednesday & Thursday & Friday \\
\hline Ward A & $\mathrm{Y}$ & 10:30-11:30 & & 9:00-10:00 & & \\
\hline Ward B & $x$ & 10:30-11:30 & & 9:00-10:00 & & \\
\hline Ward C & $\mathrm{Y}$ & 9:00-10:00 & & 10:30-11:30 & & \\
\hline Ward D & $x$ & $9: 00-10: 00$ & & 10:30-11:30 & & \\
\hline
\end{tabular}

sessions. A third researcher will be on call to cover if either of these two researchers cannot attend a given session. Any observation session that does not occur on its scheduled day will occur the following day.

Researchers retrieving information about the material used from the gel and soap dispensers will do so per a standard operating procedure. The researchers will weigh the material in the containers each Friday.

\section{Sample size}

As participation in the study is incidental, we can only estimate how many people will be involved. Based on the calculations described below, we have planned to observe participants over a long enough period to record 30-50 hand hygiene opportunities in each ward in each week. The number of participants we could expect to observe hourly on the four wards in question between the hours of 9:00 and 12:00 was estimated by the Director of Infection Protection and Control based on personal experience.

The sample size calculations are based on a control group hand hygiene compliance rate of $15 \%-20 \%$ and a clinically significant change of $15 \%$, which is consistent with the effects found in the previous ward-based studies. ${ }^{23}$ The sample size for the present study was calculated under the 'worst case' assumption that there will be a carry-over effect; that is, the effects of each condition are not independent of one another within the wards. When such carry-over exists, data from only the first observation period can be used, since each ward sees only one condition. Table 3 gives the sample size per group

Table 3 The sample size per group over the 6-week observation period to detect a significant 15\% difference at the one-tailed and two-tailed 0.05 alpha level with $80 \%$ power

\begin{tabular}{lll}
\hline & \multicolumn{2}{l}{ Carry-over exists } \\
\cline { 2 - 3 } Lower $\%$ & One sided & Two sided \\
\hline 15 & 93 & 121 \\
20 & 109 & 138 \\
\hline
\end{tabular}

over the 6-week observation period to detect a significant $15 \%$ difference at the 0.05 alpha level (one-tailed and two-tailed, respectively) with $80 \%$ power. One-tailed tests will be used to compare single prime conditions with the corresponding control conditions, whereas two-tailed tests will be used to compare the combined prime condition with the single prime conditions.

Using a sample size of 138 per group and 6 weeks of observations, it will be necessary to make at least 28 observations during each observation session in each ward in each week. We have planned to observe over long enough periods to obtain 30-50 observations in each ward each week. This will give us $93 \%$ power for a one-tailed test and $87 \%$ power for a two-tailed test.

\section{ASSIGNMENT OF CONDITIONS Allocation generation sequence}

An online tool will be used to generate a Latin squares design describing when each ward will be in each condition. $^{34}$

\section{Blinding}

It is not possible to render participants completely blind to the study. However, unless a person asks the researcher to explain why the primes are present, they will not be told. Researchers will record when people ask them to explain why they are on the ward during observation sessions on the hand hygiene observation case report forms.

\section{DATA COLLECTION}

\section{Data collection methods}

Observational sessions: During observation sessions, researchers will be placed near the entrance to each ward in full view of the gel and soap dispensers but at a discreet distance so as not to interfere with the hospital's day-to-day routines. The data will be recorded onto paper case report forms similar to those used in past hand hygiene compliance studies. ${ }^{35}$ The researchers will record each person's apparent gender (female or male), 
apparent role at the hospital (doctor, nurse, other staff or visitor) and whether she/he used the gel (yes/no) and soap (yes/no) dispensers.

The amount of product delivered from the gel and soap dispensers: During the study identification (ID), stickers will be affixed to the gel and soap containers within each relevant dispenser. The ID stickers will contain the ward number, dispenser number and container number; for example, the first container located in the dispenser nearest to the door in ward X will be labelled 'X.01.01'. Additionally, the label will inform ward-staff not to discard these containers but rather to return them to the Director of Infection Prevention and Control via the hospital's internal mail. Each Friday, a researcher will weigh the containers and record their new weight on paper case report forms. While the cross-over design of the study will facilitate comparisons between wards (with a control group present at all steps in the study), it is important to note that additional covariates will not be measured to account for other factors that affect gel and soap use, for example, variations in the amount of ward traffic over the year.

HAIs: Using hospital records, the Director of Infection Prevention and Control or an approved researcher will retrieve the information regarding HAIs that occur in hospital during the cross-over study. For the purposes of this study, a HAI is defined as an infection acquired in hospital by staff members and patients admitted for a reason other than the infection contracted and includes infections that appear in patients shortly after discharge. ${ }^{36}$ The director or an approved researcher will record: what date the infection was acquired, in what ward it was acquired, where the source data were located and what type of infection it was (blood culture, skin/soft tissue, urinary tract infection, MRSA, CDI or other). The director or an approved researcher will also retrieve the number of HAIs from the previous 10 years in these wards to indicate a base rate of expected HAIs. These HAIs will be recorded on paper case report forms.

All paper case report forms will be stored in the site file retained in a secure room by the sponsor.

\section{Data management}

The data management plan fully complies with the 1998 Data Protection Act as well as the principles of Good Clinical Practice and University policies. As a reminder, no patient-identifiable information will be collected. The paper case report forms will be stored in the site file in a secured room by the sponsor for 10 years. The data contained in the paper case report forms will be transferred to electronic files. The electronic files will be stored on a password protected file stored on a portable storage device stored in the site file and periodically backed up on to centrally secured servers. Data transfer will be restricted between collaborators through approved email accounts.

\section{Statistical methods}

Descriptive statistics will be calculated for observed individuals and their activities. These data include apparent gender, apparent role and the use of the gel and soap dispensers. Frequency and percentage scores will be calculated for each characteristic/activity by ward and phase. The statistical analysis for objectives 1-4 will be performed using Statistical Analysis System 9.3 (SAS Institute), and a 0.05 alpha level will be used to determine statistical significance. The study has been powered only for the primary outcome, and the p-values will be adjusted for multiple comparisons among the means of the four conditions using the Tukey-Kramer method.

\section{Objective 1}

Data analysis will begin with a generalised linear model ${ }^{37}$ to test for carry-over effects in the cross-over design. ${ }^{38}$ The outcome will be binary (complied with hand hygiene expectations or did not comply). The predictors will be phase, ward and the interaction of phase and ward. If the interaction term is significant, then the outcomes are not independent, carry-over has occurred and only phase 1 data can be analysed. The outcome data for hand hygiene observations are binary. If carry-over exists, then generalised linear models will be used to compare the four conditions, using phase 1 data only. Planned comparisons will be made between each of the single prime conditions and control and between each of the single prime conditions and the combined prime condition.

\section{Objective 2}

In addition to the above model, two more models will be run using the same predictor variables described above. However, the outcome variables will be the amount of gel material used and the number of soap dispenser activations.

\section{Objective 3}

The models described above will also include terms for gender or role plus an interaction term for gender or role and condition. Planned comparisons will be made between gender and role for each condition and among conditions for gender or role.

\section{Objective 4}

Under the carry-over condition, a generalised linear model for a Poisson distribution (indicating that negative binomial over-dispersion is evident) will be used to compare the proportion of HAIs under each condition. The ward census data will be used as an offset to provide a denominator for the proportion. If there is no carry-over, then a generalised linear model will be used to compare the proportion of participants that adhered to hand hygiene protocols under the various conditions specified in the hypotheses outlined above. There will be an additional term in the models to adjust the results for the variation caused by the different wards. The planned comparisons will be performed as outlined above. 


\section{Objective 5}

An economic analysis will be conducted to identify the incremental costs and outcomes of the interventions. Results will be expressed in terms of cost per additional percentage of compliance, cost per avoided HAI and cost per QALY. This will be complemented by an estimate of the costs and health benefits of rolling out the most effective intervention at the country level.

Micro-costing will first be used to estimate the intervention cost in each arm. This will involve collecting cost data for each type of prime, including fixed (eg, purchase or installation) and variable (eg, maintenance) costs, and recording product use (ie, gel or soap). We will then develop a decision-analytic model to capture the full impact of the intervention and assess long-term costs and outcomes. Intervention impact on hand hygiene compliance obtained in the study will form the starting point of the model. These effects will be translated into (1) avoided HAI and (2) long-term costs and health outcomes (ie, QALYs). The model will combine evidence from a range of sources, including study data, data from hospital records and publicly available data. We will follow recommended best practice in identifying and synthesising the evidence to inform model parameters for which data are not available from the study (eg, costs and QALY following a HAI) ${ }^{39}$ Importantly, we will follow previous work to determine the relationship between increased hand hygiene compliance and infection rates; analysis of before-after infection rates at the ward level will also be examined (see online supplementary appendix 2 for summary of data requirements)..$^{40414}$

The decision analytic model will be generated from the perspective of the provider, adopting various time horizons (eg, 1 year, 5 years and lifetime), and incorporate robustness tests predicated on varying assumptions regarding the longer-term sustainability of increased compliance. Costs and outcomes will be discounted at $3.5 \%$ per annum, and parameter uncertainty will be addressed through probabilistic sensitivity analysis. The economic evidence obtained from the model will be combined with national data on infection rates, patient flows (volume) and number of relevant providers to assess the likely costs and benefits of rolling out the intervention at the country level.

\section{MONITORING \\ Data monitoring}

After the observing researchers transfer the data contained in the paper case report forms to an electronic file, another researcher will check the accuracy of $5 \%$ of the input data. If the accuracy is less than $95 \%$, then that entire file will be collaboratively checked by both observing researchers. If the observing researchers disagree about any data that are in the case report form, then the primary investigator will arbitrate.
Harms

Plans for collecting, assessing, reporting and managing solicited and spontaneously reported adverse events and other unintended effects will follow the sponsors' pre-existing protocols.

\section{Auditing}

The sponsor does not plan to perform an audit during the study. The primary investigator will appoint a collaborator to audit the site file at its completion.

\section{ETHICS AND DISSEMINATION}

\section{Research ethics approval}

Research ethics approval was obtained from the South Central-Oxford C Research Ethics Committee (16/ SC/0554), the Health Regulatory Authority and the sponsor.

\section{Protocol amendments}

Any substantial protocol modifications, for example, changes to eligibility criteria, will be communicated to relevant collaborators via email. Within the email, collaborators will be asked to confirm that they have read the email. The principal investigator will call any collaborator who does not confirm receipt within7 calendar days.

\section{Dissemination}

We intend to publish a manuscript with the results of this study in a peer-reviewed journal. Authorship eligibility will be assessed by the primary investigator and informed by the journal's guidelines. All the present coauthors will be eligible to be coauthors on future publications, with the order determined by the primary investigator. However, should the journals not allow enough coauthors, some authors may be placed in the acknowledgements as determined by the primary investigator. On completion of the study and after publication of the primary manuscript, data requests will be handled by the lead author and primary investigator.

\section{DISCUSSION}

Poor hand hygiene remains a serious issue undermining patient safety. Whether people clean their hands on entering a ward is affected by the design of the physical environment. Hospitals already actively recognise the importance of the environment in this regard. Gel and soap dispensers are placed at every ward's entrance to ensure that people can clean their hands. ${ }^{43}$ Unfortunately, making the opportunity available has not proven sufficient to ensure compliance. Evaluating the effectiveness of environmental primes that nudge people to clean their hands on entering a ward is a promising way forward.

Most healthcare workers already know why hand hygiene is important and how to adequately clean their hands. ${ }^{44}$ However, as hospitals are busy places, it is inevitable that sometimes other activities will take priority over effective 
hand hygiene practices. ${ }^{45}$ If this happens too frequently, not cleaning one's hands may become an enduring habit. The degree to which this happens is unknown, but any degree is unfortunate. To make compliance with effective hand hygiene practices easier, hospital administrators should aim to design the environment in such a way that such practices become habitual, default behavioural patterns that are rarely forgotten. ${ }^{46}$ The primes to be investigated in this study provide a potentially powerful mechanism for achieving this fundamentally important goal.

While the present study does not attempt to uncover the theoretical mechanism that may underlie the olfactory and visual primes' effects, previous experiments have done so. The olfactory primes may activate hand hygiene via an associative mechanism, that is, the citrus smells found in many cleaning products may stimulate the desire to clean. Indeed, in a previous study, a clean smell increased the number of people who cleaned up after themselves while eating crumbly cookies. ${ }^{47}$ Regarding the visual prime, previous studies suggest that pictures of eyes may nudge people to comply with known social rules. For example, pictures of eyes similar to the picture to be deployed in the present study have been used successfully to increase the amount of money people contribute voluntarily to charity and reduce the incidence of thefts. ${ }^{48}$ 495051

Regarding the present study's limitations, it is important to note that the interventions are designated to be pragmatic, and the evaluation will take place in a real-life setting. In consequence, one practical limitation of our study is that the primes are not the only active interventions taking place at the hospital. While it would be ideal to restrict all other interventions during our study, this is not realistic. Owing to the importance of minimising HAIs, the hospital is not able to stop active in-house campaigns regarding hand hygiene; for example, signage reminding people to clean their hands and mandatory training of staff will remain in place throughout the duration of the present study. Therefore, to be significant, the interventions' effects need to be more powerful than the effects the hospital would expect based on its existing practices.

Another practical limitation is that observers will only monitor the soap and gel dispensers located near the wards' entrances. The choice to only observe at this location is a limitation because the entrances contain a very narrow subset of all the dispensers. Wards typically have dispensers at all points of care, for instance, alcohol gel dispensers are often placed outside the entrances to wards and near the bedside of each patient; in some cases, portable dispensers are carried around (eg, in people's pockets or handbags).$^{52}$ While ideally we would like to gather data from all relevant locations in each ward, such a strategy is not possible practically.

We have chosen instead to monitor the dispensers located near the wards' entrances because it allows a limited number of observers to more easily record each unique opportunity for hand hygiene complaint behaviour. Increasing the number of observers beyond this practical minimum would likely interfere with the hospital's day-to-day routines, thereby increasing the chances of a Hawthorne effect. Hawthorne effects occur when participants become aware that they are being observed and change their behaviour accordingly. ${ }^{53}$

At least two consequences of this limitation should be noted. First, our data will not capture any delayed effects of the primes; for example, if our primes increase hand hygiene complaint behaviours at patients' bedsides, this will not be detected. This limitation affects objectives 1,2 and 3 of our study.

An additional limitation of the study is that the relationship between our data and the incidence of HAIs will be incomplete. Indeed, not all HAIs are hand hygiene related. As for those HAIs that are hand hygiene related, they could arise owing to a lack of compliance beyond the confines of the ward entrances under observation. This limitation may undermine our fourth and fifth objectives.

Another limitation is that additional covariates will not be measured to account for other factors that affect gel and soap use, such as variations in the amount of ward traffic over the year. We did not add covariates about seasonal variations because a previous study has suggested that temperature variations occurring during a study performed during the same season do not affect hand hygiene behaviours. ${ }^{54}$ In addition, Larson et al found that handwashing practices did not differ significantly by season. ${ }^{55}$

A further limitation is that our primary outcome data are reliant on human observer's ratings. However, although using human observers creates a limitation, it also offers a distinct advantage over automated data collection. Specifically, human observers can count the number of opportunities for people to clean their hands in addition to whether said people clean their hands. Notably, sophisticated automatic counters are being developed that can record both opportunity and occurrence and can even give people personalised feedback. This technology is exciting but is still cost prohibitive and likely will not address the cleaning behaviours of hospital visitors. While future studies may be able to take advantage of this new technology, at present, human observers remain the most fruitful means of data collection available.

\section{Author affiliations}

${ }^{1}$ Behavioural Science Group, Warwick Business School, The University of Warwick, Coventry, UK

${ }^{2}$ Primary Care Clinical Sciences Institute of Applied Health Research, College of Medical and Dental Sciences, University of Birmingham, Birmingham, UK

${ }^{3}$ Infection Prevention and Control, Heart of England NHS Foundation Trust, Birmingham, UK

${ }^{4}$ Alliance Manchester Business School, The University of Manchester, Manchester, UK

${ }^{5}$ Department of Public Health Sciences, Division of Biostatistics, University of Miami Miller School of Medicine, Miami, Florida, USA

${ }^{6}$ Centre for Health Policy, Institute of Global Health Innovation, Imperial College London, London, UK 
${ }^{7}$ Department of Anesthesiology, University of Miami Leonard M. Miller School of Medicine, Miami, Florida, USA

Contributors The protocol was collaboratively developed by all coauthors. KAS wrote the protocol with coordinated input from all collaborators. Notably, NA and GPH substantially contributed to the redrafting of the manuscript. KA largely wrote the sample size section along with the statistical analyses for objectives 1-4. JM largely wrote the statistical analyses for objective 5 .

Funding Environmental engineering to increase hand hygiene compliance is part of the Health Foundation's Behavioural Insights Research Programme (grant no 7601). The Health Foundation is an independent charity committed to bringing about better health and health care for people in the UK. Trial sponsor: Heart of England NHS Foundation Trust.

Competing interests None declared.

Ethics approval The South Central-0xford C Research Ethics Committee (16/ SC/0554)

Provenance and peer review Not commissioned; externally peer reviewed.

Data sharing statement On completion of the study and after publication of the primary manuscript, data requests can be submitted to the lead author (KAS) or chief investigator (IV) of this protocol.

Open Access This is an Open Access article distributed in accordance with the Creative Commons Attribution Non Commercial (CC BY-NC 4.0) license, which permits others to distribute, remix, adapt, build upon this work non-commercially, and license their derivative works on different terms, provided the original work is properly cited and the use is non-commercial. See: http://creativecommons.org/ licenses/by-nc/4.0/

(C) Article author(s) (or their employer(s) unless otherwise stated in the text of the article) 2017. All rights reserved. No commercial use is permitted unless otherwise expressly granted.

\section{REFERENCES}

1. House of Commons (2009). Reducing healthcare associated infection in hospitals in England. https://www.publications. parliament.uk/pa/cm200809/cmselect/cmpubacc/812/812.pdf (retrieved 2 Feb 2017).

2. Luangasanatip N, Hongsuwan M, Limmathurotsakul D, et al. Comparative efficacy of interventions to promote hand hygiene in hospital: systematic review and network meta-analysis. BMJ 2015;351:h3728.

3. Plowman R. The socioeconomic burden of hospital acquired infection. Euro Surveill 2000;5:49-50 http://www.eurosurveillance. org/ViewArticle.aspx?Articleld=4.

4. Harbarth S, Sax H, Gastmeier P. The preventable proportion of nosocomial infections: an overview of published reports. J Hosp Infect 2003;54:258-66.

5. Marimuthu K, Pittet D, Harbarth S. The effect of improved hand hygiene on nosocomial MRSA control. Antimicrob Resist Infect Control 2014:3:34.

6. Erasmus V, Daha TJ, Brug H, et al. Systematic review of studies on compliance with hand hygiene guidelines in hospital care. Infect Control Hosp Epidemiol 2010;31:283-94.

7. Evans J, Stanovich KE. Dual-process theories of higher cognition: advancing the debate. Perspect Psychol Sci 2013;8:223-41.

8. Hofmann W, Friese M, Wiers RW. Impulsive versus reflective influences on health behavior: a theoretical framework and empirical review. Health Psychol Rev 2008;2:111-37.

9. Stanovich KE, Toplak ME. Defining features versus incidental correlates of type 1 and type 2 processing. Mind \& Society 2012;11:3-13.

10. Evans JS, Stanovich KE. Dual-process theories of higher cognition: advancing the debate. Perspect Psychol Sci 2013;8:223-41.

11. Latham GP, Piccolo RF. The effect of context-specific versus nonspecific subconscious goals on employee performance. Hum Resour Manage 2012;51:511-23.

12. Michie S, Richardson M, Johnston $M$, et al. The behavior change technique taxonomy v1 of 93 hierarchically clustered techniques. Ann Behav Med 2013;46:81-95.

13. Michie S, van Stralen MM, West R. The behaviour change wheel: a new method for characterising and designing behaviour change interventions. Implement Sci 2011;6:42.
14. Bargh JA. The ecology of automaticity: toward establishing the conditions needed to produce automatic processing effects. Am J Psychol 1992;105:181-99.

15. White KM, Jimmieson NL, Obst PL, et al. Using a theory of planned behaviour framework to explore hand hygiene beliefs at the ' 5 critical moments' among Australian hospital-based nurses. BMC Health Serv Res 2015;15:59.

16. Loveday HP, Wilson JA, Pratt RJ, et al. epic3: National evidencebased guidelines for preventing healthcare-associated infections in NHS hospitals in England recommendation SP6. 2014. http://www. journalofhospitalinfection.com/article/S0195-6701(13)60012-2/ fulltext (accessed 12 May 2017).

17. Naikoba S, Hayward A. The effectiveness of interventions aimed at increasing handwashing in healthcare workers - a systematic review. $J$ Hosp Infect 2001;47:173-80.

18. Goldmann D. System failure versus personal accountability-the case for clean hands. N Engl J Med 2006;355:121-3.

19. Sheeran P, Bosch JA, Crombez G, et al. Implicit processes in health psychology: diversity and promise. Health Psychol 2016;35:761-6.

20. Bargh JA. What have we been priming all these years? On the development, mechanisms, and ecology of nonconscious social behavior. Eur J Soc Psychol 2006;36:147-68.

21. Bargh JA, Chartrand TL. The unbearable automaticity of being. Am Psychol 1999;54:462-79.

22. Wryobeck J, Chen Y. Using priming techniques to facilitate health behaviours. Clin Psychol 2003;7:105-8.

23. Birnbach DJ, King D, Vlaev I, et al. A randomized study of environmental olfactory cues and their impact on hand hygiene behaviour in a simulated hospital environment. $J$ Hosp Infect 2013;85:79-81.

24. King D, Vlaev I, Everett-Thomas R, et al. "Priming" hand hygiene compliance in clinical environments. Health Psychol 2016;35:96-101.

25. Macleod-Glover N, Sadowski C. Efficacy of cleaning products for $C$. difficile: environmental strategies to reduce the spread of Clostridium difficile-associated diarrhea in geriatric rehabilitation. Can Fam Physician 2010;56:417-23.

26. Eckmanns T, Schwab F, Bessert J, et al. Hand rub consumption and hand hygiene compliance are not indicators of pathogen transmission in intensive care units. J Hosp Infect 2006;63:406-11.

27. Berhe M, Edmond MB, Bearman GM. Practices and an assessment of health care workers' perceptions of compliance with infection control knowledge of nosocomial infections. Am J Infect Control 2005;33:55-7.

28. American Society for Microbiology. (2003). Another U.S. airport trave hazard: dirty hands. http://www.eurekalert.org/pub_releases/200309/asfm-aua091103.php (retrieved 17 Jan 2017).

29. World Health Organization. Clinical Trials. http:www.who.int/topics/ clinical_trials/en/ (retrieved 15 Jun 2017).

30. ScentAir ScentDirect: Direct diffusion system. retrieved 22 Feb 2017: scentair.com.tr/ScentAir_ScentDirect4004_TechSpecs.pdf

31. Olszanowski M, Pochwatko G, Kuklinski K, et al. Warsaw set of emotional facial expression pictures: a validation study of facial display photographs. Front Psychol 2014;5:1516.

32. Richardson P, Goodwin A, Vine E. Research methods and design in psychology. Exeter: Shortun Press Ltd, 2011.

33. Sahay S, Panja S, Ray S, et al. Diurnal variation in hand hygiene compliance in a tertiary level multidisciplinary intensive care unit. Am $J$ Infect Control 2010;38:535-9.

34. Hamster and Wheel Retrieved 1 Mar 2016:http://hamsterandwheel. com/grids/index2d.php

35. McAteer J, Stone S, Fuller C, et al. Development of an observational measure of healthcare worker hand-hygiene behaviour: the hand-hygiene observation tool (HHOT). J Hosp Infect 2008;68:222-9.

36. World Health Organization (WHO). Prevention of hospital acquired infections: A practical guide. 2nd Edition. Available at. http://www. who.int/csr/resources/publications/whocdscsreph200212.pdf. (accessed 5 May 2017).

37. Dobson AJ, Barnett A. An Introduction to Generalized Linear Models $3^{\text {rd }}$ Ed. Boca Raton, FL: CRC Press, 2008.

38. Jones B, Kenward MG. Design and Analysis of Cross-Over Trials. $3^{\text {rd }}$ Ed. Boca Raton, FL: CRC Press, 2014

39. Saramago P, Manca A, Sutton AJ. Deriving input parameters for costeffectiveness modeling: taxonomy of data types and approaches to their statistical synthesis. Value Health 2012;15:639-49.

40. Huis A, Hulscher M, Adang E, et al. Cost-effectiveness of a team and leaders-directed strategy to improve nurses' adherence to hand hygiene guidelines: a cluster randomised trial. Int J Nurs Stud 2013;50:518-26.

41. Aiello AE, Larson EL. What is the evidence for a causal link between hygiene and infections? Lancet Infect Dis 2002;2:103-10. 
42. Pittet D, Hugonnet S, Harbarth S, et al. Effectiveness of a hospitalwide programme to improve compliance with hand hygiene. Infection Control Programme. Lancet 2000;356:1307-12.

43. Birnbach DJ, Nevo I, Barnes S, et al. Do hospital visitors wash their hands? Assessing the use of alcohol-based hand sanitizer in a hospital lobby. Am J Infect Control 2012;40:340-3.

44. Jenner EA, Fletcher BC, Watson P, et al. Discrepancy between self-reported and observed hand hygiene behaviour in healthcare professionals. J Hosp Infect 2006;63:418-22.

45. Dixit D, Hagtvedt R, Reay T, et al. Attitudes and beliefs about hand hygiene among paediatric residents: a qualitative study. BMJ Open 2012;2:e002188.

46. Dolan P, Hallsworth M, Halpern D, et al. Influencing behaviour: the mindspace way. J Econ Psychol 2012;33:264-77.

47. Holland RW, Hendriks M, Aarts H. Smells like clean spirit: nonconscious effects of scent on cognition and behavior. Psychol Sci 2005;16:689-93.

48. Bateson M, Nettle D, Roberts G. Cues of being watched enhance cooperation in a real-world setting. Biol Lett 2006;2:412-4.
49. Ernest-Jones M, Nettle D, Bateson M. Effects of eye images on everyday cooperative behavior: a field experiment. Evolution and Human Behavior 2011;32:172-8.

50. Francey $D$, Bergmüller R. Images of eyes enhance investments in a real-life public good. PLoS One 2012;7:e37397.

51. Nettle D, Nott K, Bateson M, Thieves 'Cycle. 'Cycle thieves, we are watching you': impact of a simple signage intervention against bicycle theft. PLoS One 2012;7:e51738.

52. Loveday HP, Wilson JA, Pratt RJ, et al. epic3: national evidencebased guidelines for preventing healthcare-associated infections in NHS hospitals in England. J Hosp Infect 2014;86 Suppl 1:S1-S70.

53. McCambridge J, Witton J, Elbourne DR. Systematic review of the Hawthorne effect: new concepts are needed to study research participation effects. J Clin Epidemiol 2014;67:267-77.

54. Birnbach DJ, Rosen LF, Fitzpatrick M, et al. An evaluation of hand hygiene in an intensive care unit: Are visitors a potential vector for pathogens? J Infect Public Health 2015;8:570-4.

55. Larson E, McGinley KJ, Grove GL, et al. Physiologic, microbiologic, and seasonal effects of handwashing on the skin of health care personnel. Am J Infect Control 1986;14:51-9. 